\section{Hypovitaminosis D in Systemic Sclerosis}

\section{To the Editor:}

We read with interest the articles by Dr. Braun-Moscovici, et al ${ }^{1}$ and Vacca, et $a l^{2}$ reporting a strikingly high prevalence of 25 -hydroxyvitamin $\mathrm{D}$ (25OHD) deficiency in patients with systemic sclerosis (SSc). Their remarkable findings confirm the results of our work, to our knowledge the first controlled study to demonstrate an increased prevalence of hypovitaminosis D in $\mathrm{SSc}^{3}$. The renewed interest in vitamin D in autoimmune diseases gives us the opportunity to show previously unpublished data and make some observations about this issue.

Our study comprised 60 patients and 60 matched controls ${ }^{3}$. 25OHD levels were lower in patients than in controls [median $23 \mathrm{ng} / \mathrm{ml}$ (range 3-92) and $39 \mathrm{ng} / \mathrm{ml}(14-138)$, respectively; $\mathrm{p}<0.001$ ] even after matching for season of sampling. Thirty-eight patients had 25OHD levels below 30 $\mathrm{ng} / \mathrm{ml} ; 4$ patients fell in the severe deficit range $(<10 \mathrm{ng} / \mathrm{ml}), 17$ in the insufficiency range ( $\geq 10$ and $<20 \mathrm{ng} / \mathrm{ml}$ ), and 17 in the mild hypovitaminosis range ( $\geq 20$ and $<30 \mathrm{ng} / \mathrm{ml}$ ). In 46 patients not supplied with oral calcium and vitamin D, median concentration of 25OHD was 21 (3-52) $\mathrm{ng} / \mathrm{ml}$.

The investigation of possible associations between 25OHD concentrations and disease features (limited or diffuse form, gastrointestinal involvement, cutaneous ulcers, and joint involvement) missed statistical significance. Moreover, no correlation with modified Rodnan skin score was observed. 25OHD and serum C-telopeptide of type I collagen (CTX), a well known marker of bone turnover, were negatively correlated only in patients $(\mathrm{R}=-0,32, \mathrm{p}=0.01)$. In order to verify the assumption that secondary hyperparathyroidism was the cause of CTX increase, we searched for a correlation between parathyroid hormone (PTH) and CTX. Surprisingly, analysis did not reach statistical significance, possibly in accord with the intriguing hypothesis raised by Allanore, et al of an extraosseous source of collagen metabolites in SSc. Interestingly, 25OHD tended to correlate with physical performance score assessed by the Medical Outcomes Study Short Form-36 (SF-36) questionnaire $(\mathrm{R}=0.33$, $\mathrm{p}=0.08$ ).

Braun-Moscovici, et $a l^{1}$ reported a high frequency of radiographically diagnosed calcinosis and acroosteolysis (70\% and 47\%, respectively), 2 disabling complications of SSc, and suggested a relationship with increased PTH levels. We clinically found calcinosis in $8 \%$ and acroosteolysis in $10 \%$ of patients; median PTH concentration was in the upper limit of the normal range $[68 \mathrm{pg} / \mathrm{ml}$ (range 30.5-120)]. Discrepancies in prevalence may be attributable to the greater sensitivity of hand radiographs in the detection of calcinosis and osteolysis and, given the contribution of vascular damage in the development of acroosteolysis, to administered drugs such as platelet aggregation inhibitors, calcium channel blockers, and intravenous iloprost.

Vacca, et $a l^{2}$ found a relationship between $250 H D$, disease activity, and acute-phase reactants, with a modulating role on disease course. Moreover, the reported failure of standard-dose supplementation to correct hypovitaminosis D has relevant practical consequences.

Vitamin D deficiency in SSc is potentially related to several factors. Dermal fibrous thickening with capillary damage could lead to a reduced drawing of previtamin D3 synthesized from 7-dehydrocholesterol by ultraviolet-B radiation in the epidermis; gastrointestinal involvement and malabsorption of dietary vitamin D could play an additional role; moreover, many patients with SSc experience a remarkable impairment in physical functioning, and are prone to a sedentary lifestyle and hence diminished sunlight exposure.

The discovery of vitamin $\mathrm{D}$ receptor in activated immune cells and of $1,25(\mathrm{OH})_{2} \mathrm{D}$ synthesis in activated macrophages drew increasing interest to the immunomodulating roles of vitamin D. Indeed, $1,25(\mathrm{OH})_{2} \mathrm{D}$ affects adaptive and innate immunity as well. It acts on $\mathrm{T}$ lymphocytes, modifying the Th1/Th2 balance and increasing regulatory T lymphocytes; on B lymphocytes, inhibiting antibody (and autoantibody) production; and on cells of the monocyte-macrophage lineage, preventing differentiation into dendritic cells. Additionally, $1,25(\mathrm{OH})_{2} \mathrm{D}$ enhances the innate immune system, boosting polymorphonuclear and monocytic antimicrobial capability ${ }^{4}$.

Vitamin D status of patients with SSc represents a largely undiscovered research field. An input to further investigations might result from recent research concerning rheumatoid arthritis (RA) ${ }^{5}$, systemic lupus erythematosus (SLE) ${ }^{6,7}$, and undifferentiated connective tissue disease (UCTD) ${ }^{8}$. A higher risk of developing RA was associated with low dietary vitamin D intake, despite subsequently published contrasting results ${ }^{5}$. Low circulating $25 \mathrm{OHD}$ was also linked to increased disease flares ${ }^{4}$. Several studies reported lower 25OHD levels in patients with SLE compared with controls and, although some results were contradictory, a correlation with disease activity scores was found ${ }^{7}$. Recently, Zold, et al achieved similar results investigating 25OHD concentration in UCTD; they also linked extremely low levels of 25OHD to development toward a definite $\mathrm{CTD}^{8}$.

Only 2 previous works investigated 25OHD levels in SSc: one was uncontrolled and did not find subnormal $25 \mathrm{OHD}$ levels ${ }^{9}$, the second comprised 19 patients and controls, and did not find differences ${ }^{10}$.

Given the most recent findings, we consider further research would be clinically important to elucidate the causes of hypovitaminosis D in SSc, its relevance to disease progression, its influence on bone metabolism and immune functions, and the potential effects of supplementation.

\section{GILBERTO CALZOLARI, MD; VALERIA DATA, MD; RENATO} CARIGNOLA, MD; ALBERTO ANGELI, MD, SCDU Medicina Interna I, Dipartimento di Scienze Cliniche e Biologiche, Università di Torino, Regione Gonzole 10, Orbassano-Turin, 10043, Italy. Address correspondence to Dr. Calzolari; E-mail: gilberto983@gmail.com

\section{REFERENCES}

1. Braun-Moscovici Y, Furst DE, Markovits D, Rozin A, Clements PJ, Nahir AM, et al. Vitamin D, parathyroid hormone, and acroosteolysis in systemic sclerosis. J Rheumatol 2008;35:2201-5.

2. Vacca A, Cormier C, Piras M, Mathieu A, Kahan A, Allanore Y. Vitamin D deficiency and insufficiency in 2 independent cohorts of patients with systemic sclerosis. J Rheumatol 2009;36:1924-9.

3. Dovio A, Data V, Carignola R, Calzolari G, Vitetta R, Ventura M, et al. Circulating osteoprotegerin and soluble RANK ligand in systemic sclerosis. J Rheumatol 2008;35:2206-13.

4. Arnson Y, Amital H, Shoenfeld Y. Vitamin D and autoimmunity: new aetiological and therapeutic considerations. Ann Rheum Dis 2007;66:1137-42.

5. Merlino LA, Curtis J, Mikuls TR, Cerhan JR, Criswell LA, Saag KG. Vitamin D intake is inversely associated with rheumatoid arthritis: results from the Iowa Women's Health Study. Arthritis Rheum 2004;50:72-7.

6. Kamen DL, Cooper GS, Bouali H, Shaftman SR, Hollis BW, Gilkeson GS. Vitamin D deficiency in systemic lupus erythematosus. Autoimmun Rev 2006;5:114-7.

7. Ruiz-Irastorza G, Egurbide MV, Olivares N, Martinez-Berriotxoa A, Aguirre C. Vitamin D deficiency in systemic lupus erythematosus: prevalence, predictors and clinical consequences. Rheumatology 2008;47:920-3.

8. Zold E, Szodoray P, Gaal J, Kappelmayer J, Csathy L, Gyimesi E, et al. Vitamin D deficiency in undifferentiated connective tissue disease. Arthritis Res Ther 2008;10:R123.

9. Serup J, Hagdrup H. Vitamin D metabolites in generalized scleroderma. Evidence of a normal cutaneous and intestinal supply with vitamin D. Acta Derm Venereol 1985;65:343-5.

10. Matsuoka LY, Dannenberg MJ, Wortsman J, Hollis BW, Jimenez SA, Varga J. Cutaneous vitamin D3 formation in progressive systemic sclerosis. J Rheumatol 1991;18:1196-8.

J Rheumatol 2009;36:12; doi:10.3899/jrheum.090439 